\title{
Contribution of nucleosome binding preferences and co-occurring DNA sequences to transcription factor binding
}

\author{
Ximiao He${ }^{1}$, Raghunath Chatterjee ${ }^{1}$, Sam John ${ }^{2,6}$, Hector Bravo ${ }^{5}$, B K Sathyanarayana ${ }^{3}$, Simon C Biddie ${ }^{2}$, \\ Peter C FitzGerald ${ }^{4}$, John A Stamatoyannopoulos ${ }^{6}$, Gordon L Hager ${ }^{2}$ and Charles Vinson ${ }^{1^{*}}$
}

\begin{abstract}
Background: Chromatin plays a critical role in regulating transcription factors (TFs) binding to their canonical transcription factor binding sites (TFBS). Recent studies in vertebrates show that many TFs preferentially bind to genomic regions that are well bound by nucleosomes in vitro. Co-occurring secondary motifs sometimes correlated with functional TFBS.

Results: We used a logistic regression to evaluate how well the propensity for nucleosome binding and cooccurrence of a secondary motif identify which canonical motifs are bound in vivo. We used ChIP-seq data for three transcription factors binding to their canonical motifs: $\mathrm{c}$-Jun binding the AP-1 motif (TGA /GTCA), GR (glucocorticoid receptor) binding the GR motif (G-ACA--- /CGT-C), and Hoxa2 (homeobox a2) binding the Pbx (Pre-B-cell leukemia homeobox) motif (TGATTGAT). For all canonical TFBS in the mouse genome, we calculated intrinsic nucleosome occupancy scores (INOS) for its surrounding 150-bps DNA and examined the relationship with in vivo TF binding. In mouse mammary 3134 cells, c-Jun and GR proteins preferentially bound regions calculated to be well-bound by nucleosomes in vitro with the canonical AP-1 and GR motifs themselves contributing to the high INOS. Functional GR motifs are enriched for AP-1 motifs if they are within a nucleosome-sized 150-bps region. GR and Hoxa2 also bind motifs with low INOS, perhaps indicating a different mechanism of action.

Conclusion: Our analysis quantified the contribution of INOS and co-occurring sequence to the identification of functional canonical motifs in the genome. This analysis revealed an inherent competition between some TFs and nucleosomes for binding canonical TFBS. GR and c-Jun cooperate if they are within 150-bps. Binding of Hoxa2 and a fraction of GR to motifs with low INOS values suggesting they are not in competition with nucleosomes and may function using different mechanisms.
\end{abstract}

Keywords: TFBS, Nucleosome, GR, c-Jun

\section{Background}

Gene expression is ultimately controlled by the DNA sequence of the genome. The dramatically different DNA composition of proximal promoters in mammals [1] compared to yeast [2] and Drosophila [3] suggests that different mechanisms regulate gene expression in those organisms. Proximal promoters in yeast and Drosophila are AT rich and tend to be poorly-bound by

\footnotetext{
* Correspondence: Vinsonc@mail.nih.gov

'Laboratory of Metabolism, National Cancer Institute, National Institutes of Health, Room 3128, Building 37, 37 Convent Drive, Bethesda, MD 20892, USA Full list of author information is available at the end of the article
}

nucleosomes both in vitro and in vivo [4-6] allowing easy access for transcription factors (TFs). In contrast, vertebrate promoters are often GC rich [7] and are well bound by nucleosomes in vitro $[4,6,8]$. In vivo, however, the GC rich promoters are instead bound by transcription factors and RNA polymerase II [4,5,9]. This observation lends support to a dynamic equilibrium switch mechanism where the promoter shifts from being bound by a nucleosome to being bound by TFs $[3,10,11]$. The kinetic interplay between these two states is mediated by the chromatin remodeling factors that disrupt, unwrap, and/or displace nucleosomes [10,12-14]. An extension of

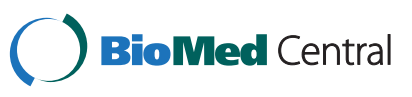


this competition model is a collaborative competition model where two TFs can bind to DNA independently but together can cooperate and displace a nucleosome if the two TFBSs are within 150-bps of each other $[10,11,15-18]$. The mechanistic details of this switch are complex with some TF being able to bind to DNA also bound by the histone octamer [19].

The determination of the in vitro binding of chicken nucleosomes to yeast genomic DNA allowed development of a scoring system that give an intrinsic nucleosome occupancy score (INOS) that indicates how well a nucleosome would bind any 150-bps of DNA $[4,6]$. This scoring system predicts that nucleosomes would bind CpG-rich regions well, which is consistent with what was observed $[5,8]$ indicates credibility to the accuracy of the calculation. A general conclusion is that nucleosomes preferentially bind cytosine and guanine, sequences that often occur in clusters called $\mathrm{CpG}$ islands in mammalian genomes [5]. Hughes and colleagues have shown that in human samples, TF binding and DNase I hypersensitive sites (DHS) preferentially localize in genomic regions with high INOS [5].

In this study, we have focused on three TFs binding to their canonical TFBSs: c-Jun binding the AP-1 motif $\left(\mathrm{TGA}^{\mathrm{C}} / \mathrm{G}_{\mathrm{GCA}}\right.$ ), glucocorticoid receptor (GR) binding the GR-like split 8-mer (G-ACA---TGT-C) [20-22], and Hoxa2 binding the homeobox Pbx motif (TGATTGAT) [23]. We show that nucleosomes are calculated to bind preferentially to both the GR and c-Jun motifs revealing an inherent competition between nucleosome and TF for binding. In contrast, the Hoxa2 motif is calculated to be less well bound by nucleosomes suggesting they are not in competition for binding the canonical motif [24]. Some Hoxa2 and GR, but not c-Jun, bound motifs have low INOS suggesting a second class of motifs that are not in competition with nucleosomes and may function using different mechanisms.

We used a logistic regression to evaluate the significance of these correlative observations and determined how well INOS and co-occurring DNA motifs could predict if a canonical motif would be bound by a TF. High INOS for canonical AP-1 motifs was a good predictor of c-Jun binding but co-occurring sequences was not predictive. For GR, in contrast, INOS was less predictive but co-occurring cis-motifs, (e.g., AP-1 or E-Box) was more predictive.

\section{Results}

GR and c-Jun proteins preferentially bind canonical DNA motifs in regions with high INOSs

Previous work has shown that dexamethasone induced GR protein binding preferentially occurs in DHS in the genome [20,21]. GR ChIP-seq data identified peaks for GR binding that were examined using MEME [25], DNA motif finding tools, and presented a position weight matrix for the enriched GR motif and the co-occurring AP-1 motif. We have extended this analysis and examined all DNA 8-mers in the form of the GR motif $(\mathrm{N}-\mathrm{NNN}-\mathrm{NNN}-\mathrm{N})$ termed a GR-like split 8-mer and calculated the enrichment of split-8-mers in GR peaks (Additional file 1: Figure S1A). Two sequences (G-ACA---TGT-C and G-ACA---CGT-C), which occur 27,176 and 7,394 times in the masked genome, are the most enriched ( 20-fold) split 8-mers in the GR peaks. To exclude repetitive parts of the genome, we focused on the masked genome comprising $\sim 55 \%$ of the genome [26]. Similar results are obtained when we examine the whole genome. The CG containing GR motif is not prominently seen in the published position weight matrix [21] reflecting it is rare in the genome. The variable enrichment of distinct sequences reflects the importance of studying individual sequence motifs instead of position weight matrices $[27,28]$. We also determined the enrichment of all 8-mers in the AP-1-like form (NNNNNNNN) in the 20,391 c-Jun peaks - the four most enriched 8-mers contained the canonical AP-1 7mer $\left(\mathrm{TGA}^{\mathrm{C}} / \mathrm{G} \mathrm{TCA}\right.$ ) (Additional file 1: Figure S1B). INOS averages for GR peaks, c-Jun peaks, and DHSs spanning a 1,500-bp region have a maximum at the center of the peak with widths of $~ 300$-bps (Figure 1A; Additional file 1: Figure S2) as shown previously for other mammalian transcription factors [5].

One way to evaluate how predictive high INOS is in TF localization in the genome would be to calculate an INOS for each nucleotide in the genome. However, to simplify the analysis, we calculated the INOS for all canonical motifs and compared this to ChIP-seq data that identify bound canonical motifs. Over half of the 20,391 c-Jun ChIP-seq peaks in mouse mammary 3134 cells contain a canonical 7-bp AP-1 motif. The average INOS across 1,500-bps for the c-Jun peaks with and without an AP-1 motif are similar (Additional file 1: Figure S3). The bound AP-1 motifs have significantly higher INOS values ( 0.7) compared to unbound motifs $(\sim 0.1)$ (Figure 1B, Additional file 1: Figure S2) $\left(\mathrm{p}<2.2 \times 10^{-16}\right)$. Figure $1 \mathrm{C}$ presents a histogram summarizing INOS distribution for all AP-1 motifs in the genome and the percentage in c-Jun ChIP-seq peaks. c-Jun preferentially binds AP-1 motifs with high INOS, while only a few AP1 motifs with low INOS are bound. $4.4 \%$ of canonical AP-1 motifs are bound by c-Jun, while up to $\sim 30 \%$ of AP-1 motifs with high INOS (from 1.8 to 2.1 ) are bound by c-Jun (Figure 1B-C). To evaluate the significance of INOS for determining c-Jun binding to the AP-1 motif, we used a logistic regression. The percent of variance explained (PVE) and area under ROC curve (AUC) by INOS for c-Jun binding canonical motifs are $10.3 \%$ and 0.76 (Table 1, Additional file 1: Table S1A) indicating 

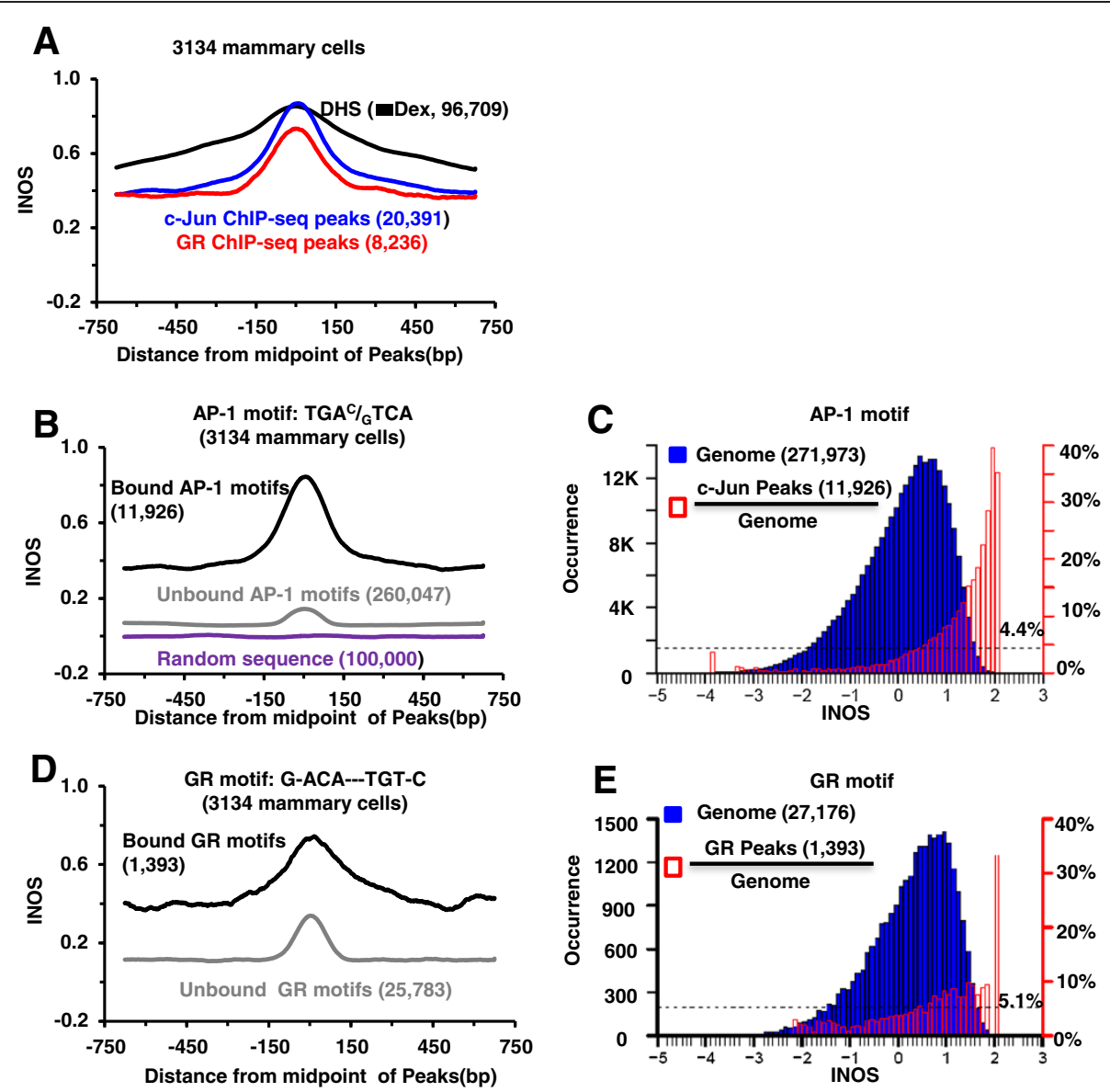

Figure 1 Intrinsic nucleosome occupancy scores for regulatory sites. (A) Average intrinsic nucleosome occupancy score (INOS) for 96,709 DNase I hypersensitive sites, 20,391 c-Jun peaks, and 8,236 GR peaks within \pm 750 -bps from the center of the peak. (B) Average INOS near ( \pm 750 -bps) canonical AP-1 motifs (TGA / $/ \mathrm{TCA}) ; 11,926$ bound and 260,047 unbound AP-1 motifs are shown. 100,000 randomly selected sequences are shown as control. (C) The blue histogram shows the distribution of INOS for all 271,973 AP-1 motifs in the mouse genome and the red histogram shows the percent bound by c-Jun indicating preference for binding to the motifs with higher INOSs. 4.4\% of all AP-1 sites are bound by c-Jun (black dashed line). (D) Average INOS near ( $\pm 750-$ bps) canonical GR motifs (G-ACA--TGT-C); 1,393 bound and 25,783 unbound GR motifs are shown. (E) GR binds preferentially to GR motifs with higher INOSs. 5.1\% of all GR sites are bound by GR (black dashed line).

INOS is predictive of c-Jun binding. When we examine c-Jun binding to non-AP-1 sequences, INOS is less predictive (Additional file 1: Table S2A-B).

The INOS for all canonical GR motifs (G-ACA--TGT-C) in the genome were also determined (Figure 1D, Additional file 1: Figure S2C). Like c-Jun, both peak and background INOS values are higher for the bound motifs than the unbound GR motifs (Figure 1D) $\left(\mathrm{p}<2.2 \times 10^{-16}\right)$ and have higher INOS near the peak ( $\pm 150 \mathrm{bps}$ ) compared to nearby ( \pm 750 to $\pm 150 \mathrm{bps}$ ). GR binds $5.1 \%$ of the canonical GR motifs, and preferentially binds to motifs in the genomic regions with higher INOSs (Figure 1E). Unlike c-Jun, some GR motifs with low INOS $(-2$ to -1$)$ are bound (Figure 1C, Figure 1E, Additional file 1: Figure S2E) which may be indicative of a non-competitive model for TF and nucleosome binding [29]. Using the logistic regression, the PVE and AUC for INOS in the peaks to GR binding are $2.9 \%$ and 0.63 , much less than $10.3 \%$ and 0.76 for c-Jun (Table 1, Additional file 1: Table S1A-B).

Two additional INOS parameters are predictive of GR and c-Jun binding to canonical motifs

In addition to the INOS calculated when the canonical motif is at the center of the nucleosome-sized 150-bp genomic region (Peak value), we also examined the 'Background' value of INOS ( \pm 750 to \pm 150 bps) as well as the 'Peak height' or 'Relative Peak' value of INOS (the difference between the Peak and Background values) [5] [30] (Figure 1A). Additional file 1: Table S3A characterizes AP-1 motifs based on 'Background' and 'Relative Peak' values revealing how these parameters can predict which motifs will be bound by c-Jun. For example, $14.4 \%$ of the 2,184 AP-1 motifs with "high" 'Background' and 'Relative Peaks' values are bound by c-Jun while zero of the 2,204 AP-1 sites with "low" 'Background' and 
Table 1 Modeling c-Jun, GR and Hoxa2 binding using a logistic regression

\begin{tabular}{|c|c|c|c|c|c|c|c|c|c|}
\hline \multirow[t]{3}{*}{ Evaluated parameters } & \multicolumn{3}{|c|}{ AP-1 motif } & \multicolumn{3}{|c|}{ GR motif } & \multicolumn{3}{|c|}{ Pbx motif } \\
\hline & \multicolumn{3}{|c|}{$(\mathrm{TGA} / \mathrm{G} T C A)(11,926 / 271,973)$} & \multicolumn{3}{|c|}{$($ (G-ACA-TGT-C) $(1,393 / 27,176)$} & \multicolumn{3}{|c|}{ (TGATTGAT) $(638 / 59,802)$} \\
\hline & PVE (\%) & CV err. & AUC & PVE (\%) & CV err. & AUC & PVE (\%) & CV err. & AUC \\
\hline A1. Peak & 10.3 & 0.04 & 0.76 & 2.9 & 0.05 & 0.63 & 0.5 & 0.01 & 0.57 \\
\hline A2. Background & 6.5 & 0.04 & 0.70 & 3.0 & 0.05 & 0.64 & 0.1 & 0.01 & 0.50 \\
\hline A3. Relative peak & 1.9 & 0.04 & 0.62 & 0.2 & 0.05 & 0.53 & 0.6 & 0.01 & 0.57 \\
\hline A. INOS $(\mathbf{A} 1+\mathbf{A} 2+\mathbf{A} 3)$ & 11.5 & 0.04 & 0.77 & 3.9 & 0.05 & 0.66 & 0.6 & 0.01 & 0.57 \\
\hline B. Overlap with CGIs & 0.9 & 0.04 & 0.53 & 0.1 & 0.05 & 0.51 & 0.0 & 0.01 & 0.51 \\
\hline C. Cluster of canonical motifs & 5.5 & 0.04 & 0.66 & 0.6 & 0.05 & 0.51 & 0.1 & 0.01 & 0.51 \\
\hline D. Co-occurrence of 2 nd motif* & 0.2 & 0.04 & 0.51 & 2.6 & 0.05 & 0.57 & 0.1 & 0.01 & 0.51 \\
\hline$A+B$ & 12.3 & 0.04 & 0.78 & 3.9 & 0.05 & 0.66 & 0.6 & 0.01 & 0.57 \\
\hline$A+B+C$ & 13.9 & 0.04 & 0.79 & 4.2 & 0.05 & 0.67 & 0.7 & 0.01 & 0.58 \\
\hline $\mathrm{A}+\mathrm{B}+\mathrm{C}+\mathrm{D}$ & 14.0 & 0.04 & 0.79 & 6.7 & 0.05 & 0.70 & 0.8 & 0.01 & 0.58 \\
\hline
\end{tabular}

Modeling the c-Jun binding to AP-1 motif (TGA $/ \mathrm{G} T C A)$, GR binding to GR motif (G-ACA-TGT-C), Hoxa2 binding to Pbx motif (TGATTGAT) were performed by the logistic regression using generalized linear models (GLM). PVE Percent of variance explained (\%) = 1-(deviance/null.deviance). CV err. estimated 11-fold crossvalidation prediction error, AUC Area under the ROC curve. *The Co-occurrence of 2nd motifs: the co-occurrence motif of AP-1 motif is GR motif (G-ACA-TGT-C), the co-occurrence of GR motif is AP-1 motif (TGA $/ \mathrm{G} T C A)$, the co-occurrence of Pbx motif is GR-like 8-mer (G-TGA-ATG-C). The PVEs of INOS and Co-occurrence of 2nd motif for AP-1, GR and Pbx motif are shown in bold to highlight the difference between the motifs.

'Relative Peaks' values are bound. To extend the insights gained from averaging the INOS for all motifs, we examined individual values of 'Relative Peaks'. $77 \%$ of bound AP-1 motifs have higher INOS near the motif ( \pm 150 bps) compared to adjacent DNA sequences ( \pm 750 to \pm 150 bps) while only $59 \%$ of unbound canonical AP-1 motifs have this trait $\left(\mathrm{p}<2.2 \times 10^{-16}\right)$. Similar but less dramatic results are observed for GR localization to canonical GR motifs (Additional file 1: Table S3B). 74\% of canonical GR motifs have a higher INOS near the motif $( \pm 150$ bps) compared to background ( \pm 750 to $\pm 150 \mathrm{bps}$ ), while $66 \%$ of the unbound canonical GR motifs have this trait $\left(\mathrm{p}=8.9 \times 10^{-10}\right)$.

A logistic regression indicates that the percent of variance explained (PVE) and AUC for 'Background' INOS for c-Jun localization to the canonical motif are $6.5 \%$ and 0.70 , while the PVE and AUC of 'Relative Peaks' INOSs for c-Jun binding are only $1.9 \%$ and 0.62 (Table 1, Additional file 1: Table S1A). The least predictive factor was the relative peak value, which was previously reported as supporting a collaborative competition model [5,30]. For c-Jun binding to AP-1 canonical motifs, the combined PVE and AUC for these three parameters are $11.5 \%$ and 0.77 (Table 1, Additional file 1: Table S1A). For GR localization to canonical motifs, the PVE and AUC for Background INOSs are $3.0 \%$ and 0.64 but the PVE and AUC of Relative Peak INOSs are only $0.2 \%$ and 0.53 with the PVE and AUC of all three parameters are $3.9 \%$ and 0.66 (Table 1, Additional file 1: Table S1B).
Nucleosomes preferentially bind GR and AP-1 motifs but not the Pbx motif

c-Jun and to an extent GR, prefer to bind motifs embedded in 150-bp long nucleosome-sized regions with high INOS, however the contribution of the TFBS itself to INOS of the entire 150-bps remains unclear. To address this issue, for each motif, we calculated the INOS for 1,000 simulated random 150-bp DNA sequences with $42 \%$ GC content as occurs in the mouse genome with the motif at the center. We examined all 32,896 GR-like 8-mers (N-NNN-NNN-N) and AP-1-like 8-mers (NNNNNNNN), as well as a control set where motifs at the center were also randomized (Figure 2A-B). The canonical GR motif (G-ACA--- ${ }^{\mathrm{T}} / \mathrm{C}$ GT-C) has a higher score than the average INOS or the control set $(\mathrm{p}=1.5 \times$ $10^{-33}$ ) suggesting that nucleosomes preferentially bind the GR motif. When we examined continuous 8-mers, a distribution was observed with many classic motifs, including AP-1, having higher INOS than random, similar to what was observed for GR (Figure 2B).

We next used the logistic regression to evaluate the contribution of the 150-bps without the TFBS to INOS. To examine this, we maintained the DNA sequences surrounding the motif and replaced the motif by random bases with GC content of $42 \%$, and then calculated INOS. Excluding the TFBS from the calculation of INOS decreases the PVE of INOSs from $10.3 \%$ to $7.4 \%$ and AUC from 0.76 to 0.72 for c-Jun localization indicating that both the AP-1 motif and additional sequences drive preferential nucleosome binding (Additional file 1: Table S1A). Similar results (the PVE decreases from $2.9 \%$ to $2.7 \%$, AUC 
A GR-like split-8mer: N-NNN---NNN-N
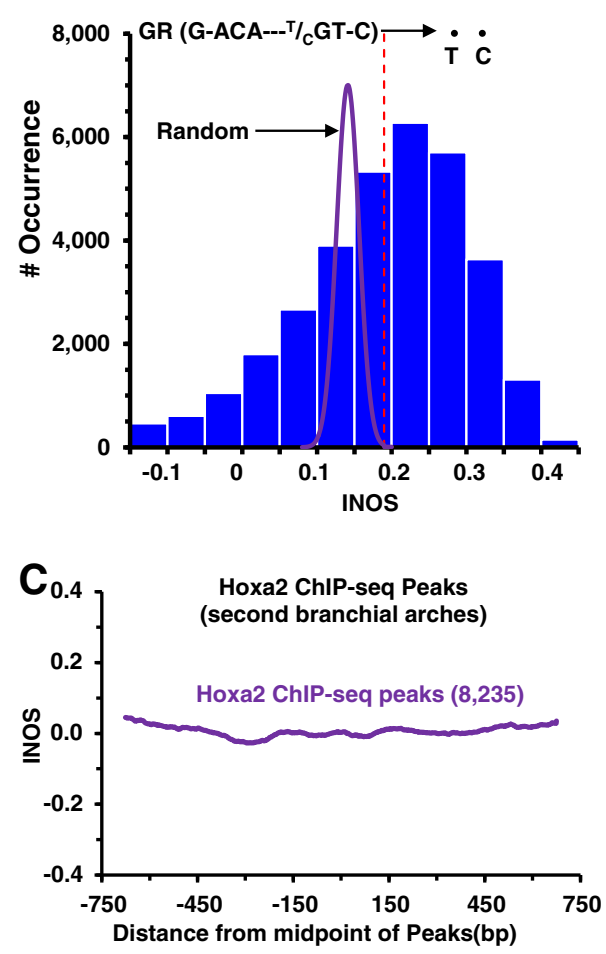

E

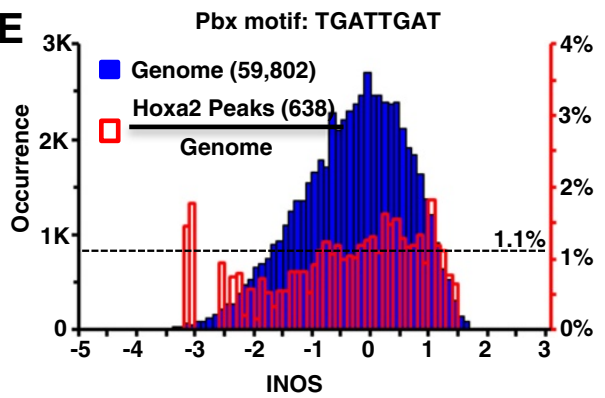

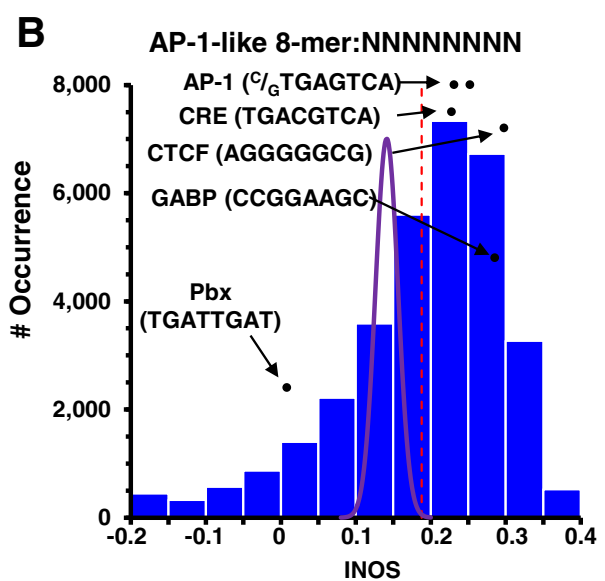

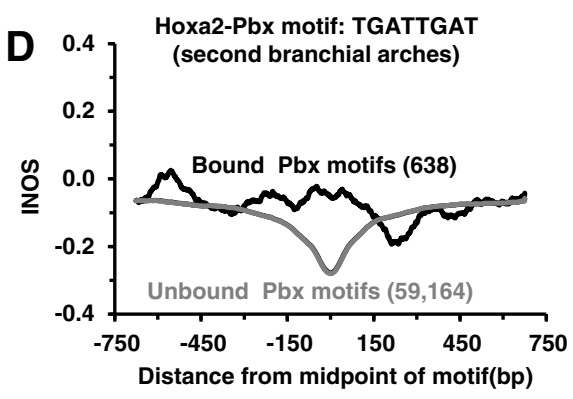

Figure 2 The GR and AP-1 motifs are calculated to be well-bound by nucleosomes. (A) Distribution of the INOS for 32,896 GR-like 8-mers (N-NNN---NNN-N). The INOS for each split 8-mer is calculated from 1,000 simulated 150-bp DNA sequences with the GR-like 8-mer in the center. The GR motifs are calculated to bind nucleosome better than the average GR-like 8-mer. The two GR motifs (G-ACA--- $/$ /CTT-C) are highlighted. Average INOS for all split 8-mers is shown as a dashed vertical red line. Distribution of INOS $(0.141 \pm 0.015)$ for 32,896 random control set following Gaussian distribution is shown in purple. (B) Distribution of the INOS for all continuous 8-mers (NNNNNNNN). (C) Average INOS for 8,235 Hoxa2 ChIP-seq peaks [23]. (D) Average INOS near ( $\pm 750-$-bps) canonical Pbx motifs (TGATTGAT); 638 bound as observed in the Hoxa2 ChIP-seq peaks and 59,164 unbound Pbx motifs are shown. (E) The blue histogram shows the distribution of INOS for all 59,802 Pbx motifs and the red histogram shows the percent bound by Hoxa2.

decreases from 0.633 to 0.632 ) were observed for GR motifs (Additional file 1: Table S1B).

Some TFBSs, such as Homeobox Pbx (TGATTGAT) motif, have low INOS $\left(\mathrm{p}=4.1 \times 10^{-21}\right)$ and thus are not competing with nucleosomes for binding in vivo (Figure 2B-C). Examining published Hoxa2 ChIP-seq peaks from mouse secondary bronchial arches [23] (Figure 2C), we observed that motifs with both high and low INOS were bound. We examined all 59,802 occurrence of the $\mathrm{Pbx}$ motif in the genome and calculated INOSs for both the 638 bound and 59,164 unbound Pbx motifs (Figure 2D-E, Additional file 1: Figure S1C). The bound TFBS with low INOS suggest the homeobox protein is not in competition with nucleosomes for binding to DNA as previously observed for TFs that bind yeast promoters [29].

\section{GR binding and open chromatin}

We correlated GR binding with the presence of a DHS to better understand the relationship between TF binding, the presence of a canonical motif, and nucleosome remodeling. 
GR peaks were classified into three groups, i.e. $71 \%$ in preprogrammed DHSs (DHSs observed in the absence of dexamethasone), $24 \%$ in re-programmed DHS (new DHSs induced after activation of GR by dexamethasone), and the remaining $5 \%$ in the regions that are not DHSs, which we term un-programmed DHS. GR motifs in all three groups have similar INOS with the pre-programmed DHS having slightly higher values (Additional file 1: Figure S2A and S2C) $9 \%$ of the GR peaks in pre-programmed DHSs contain a canonical GR motif, $34 \%$ of GR peaks at reprogrammed DHSs contain a canonical GR motif, while $48 \%$ of GR peaks not in DHSs contain a canonical GR motif (Table 2, Additional file 1: Figure S2A and S2C), suggesting a canonical motif facilitates GR binding in chromatin $\left(\mathrm{p}<2.2 \times 10^{-16}\right)$. Inclusion of the experimentally determined DHSs to the logistic regression, increased the PVE for c-Jun and GR binding to $56.5 \%$ and $39.3 \%$ respectively, and increased AUC to 0.96 and 0.88 (Additional file 1: Table S1A-B).

To more clearly examine GR binding in the unprogrammed peaks, we compared the sequencing tagdensity of GR and c-Jun binding, and the DHS signal within 3-Kb of the peak (Figure 3A-B). In the 425 unprogrammed GR peaks, a clear signal for GR binding was observed yet there was a less signal for the DHS. In contrast to $\mathrm{GR}$, more than $95 \% \mathrm{c}$-Jun binding is at pre-programmed DHSs. Only $1.5 \%$ of c-Jun binding is in re-programmed DHSs $(n=321)$ and in all these cases, a clear signal of tag density is observed for c-Jun, GR and DHS (Figure 3B). $42 \%$ of c-Jun peaks in re-programmed DHSs have a canonical AP-1 motif and 93\% are bound by GR suggesting these two proteins are acting together resulting in more open chromatin. 3\% of c-Jun peaks are in un-programmed DHSs but little tag density is observed for either c-Jun or DHSs in contrast to the GR tag density at un-programmed peaks suggesting that GR has a higher propensity to bind in chromatin (Figure 3B, Additional file 1: Figure S2B, Figure S2D).

An alternative method to evaluate GR and c-Jun binding is to compare normalized tag density within 150-bps for GR and c-Jun peaks containing canonical motifs with DHS reads (Additional file 1: Figure S4A-B). We examined the AP-1 8-mer (ATGAGTCA) for a more accurate comparison with the GR motif 8-mer (G-ACA--TGT-C). The slope of $\mathrm{c}$-Jun reads against DHS reads at the non-DHS regions is much higher than for GR implying GR can bind to the non-DHS regions better than c-Jun. Visualization of tag-density profiles within the $3-\mathrm{Kb}$ upstream and downstream of the canonical GR motifs and AP-1 8-mer (ATGAGTCA) supports GR binding in non-DHS regions (Additional file 1: Figure S4C-D). X-ray crystal structures of GR (PDB ID: 1R4O) and AP-1 (PDB ID: 1FOS) proteins bound to their canonical DNA motifs were overlaid with an X-ray structure of the histone octamer (PDB ID: 1AOI) bound to DNA (a nucleosome) using the program Chimera [31] to create a physical model of these proteins bound to the same DNA. No physical clashes were observed when GR and the histone octomer bind the same DNA as has been experimental shown [19], while clashes are observed when both AP-1 and the histone octamer bind the same DNA (Figure 3C).

Table 2 Effect of A-FOS on GR binding to the GR motif and 1-bp variants

\begin{tabular}{|c|c|c|c|c|c|}
\hline GR Motifs & $\begin{array}{l}\text { \# in } \\
\text { Genome }\end{array}$ & $\begin{array}{l}\text { \# in Peaks } \\
\text { (\% of Peaks with the Motif) }\end{array}$ & $\begin{array}{l}\% \text { With } \\
\text { AP-1 (\% in c-Jun Peaks) }\end{array}$ & $\begin{array}{l}+ \text { AP-1 } \\
\% \text { A-Fos }\end{array}$ & $\begin{array}{l}\text { - AP-1 } \\
\% \text { A-Fos }\end{array}$ \\
\hline & & \multicolumn{4}{|l|}{ Pre-Programmed (5,865 Peaks) } \\
\hline G-ACA-TGT-C & 27,176 & $523(9 \%)$ & $23 \%(80 \%)$ & $44 \%$ & $15 \%$ \\
\hline G-ACA-CGT-C & 7,394 & $131(2 \%)$ & $23 \%(80 \%)$ & $38 \%$ & $20 \%$ \\
\hline \multirow[t]{2}{*}{ 1-bp Variants } & 575,842 & 1,719 (30\%) & $31 \%(86 \%)$ & $54 \%$ & $28 \%$ \\
\hline & & \multicolumn{4}{|l|}{ Re-Programmed (1,946 Peaks) } \\
\hline G-ACA-TGT-C & 27,176 & $665(34 \%)$ & $14 \%(44 \%)$ & $45 \%$ & $9 \%$ \\
\hline G-ACA-CGT-C & 7,394 & $134(7 \%)$ & $10 \%(23 \%)$ & $39 \%$ & $4 \%$ \\
\hline \multirow[t]{2}{*}{ 1-bp Variants } & 575,842 & $806(41 \%)$ & $18 \%(33 \%)$ & $50 \%$ & $11 \%$ \\
\hline & & \multicolumn{4}{|l|}{ Un-Programmed (425 Peaks) } \\
\hline G-ACA-TGT-C & 27,176 & $205(48 \%)$ & $6 \%(0 \%)$ & $58 \%$ & $8 \%$ \\
\hline G-ACA-CGT-C & 7,394 & $29(7 \%)$ & $7 \%(0 \%)$ & $50 \%$ & $4 \%$ \\
\hline 1-bp Variants & 575,842 & 186 (44\%) & $9 \%(6 \%)$ & $41 \%$ & $11 \%$ \\
\hline
\end{tabular}




\section{A GR peaks} $(8,236)$

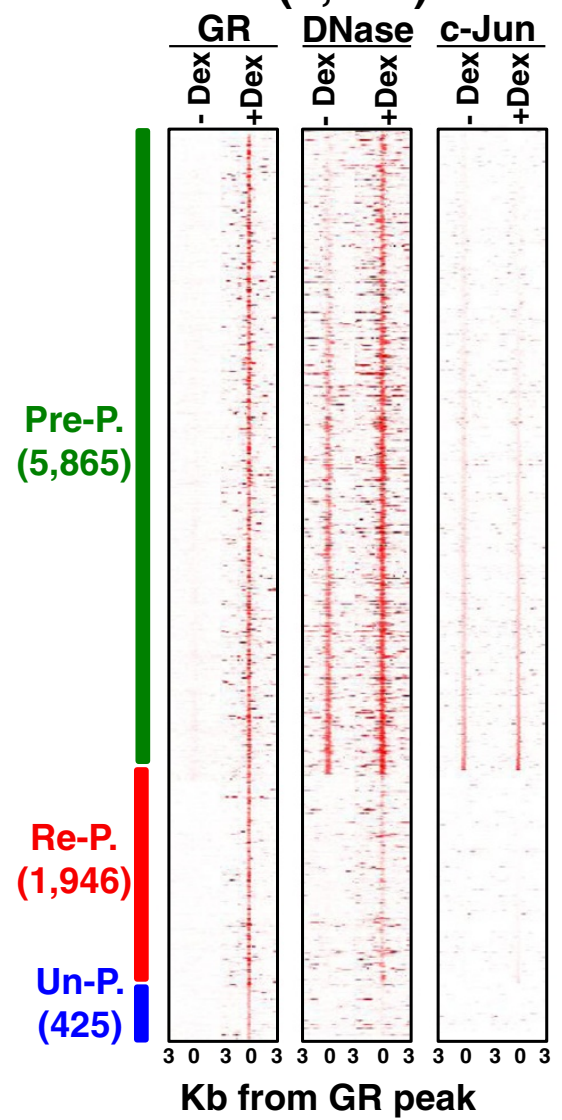

B C-Jun peaks $(20,391)$

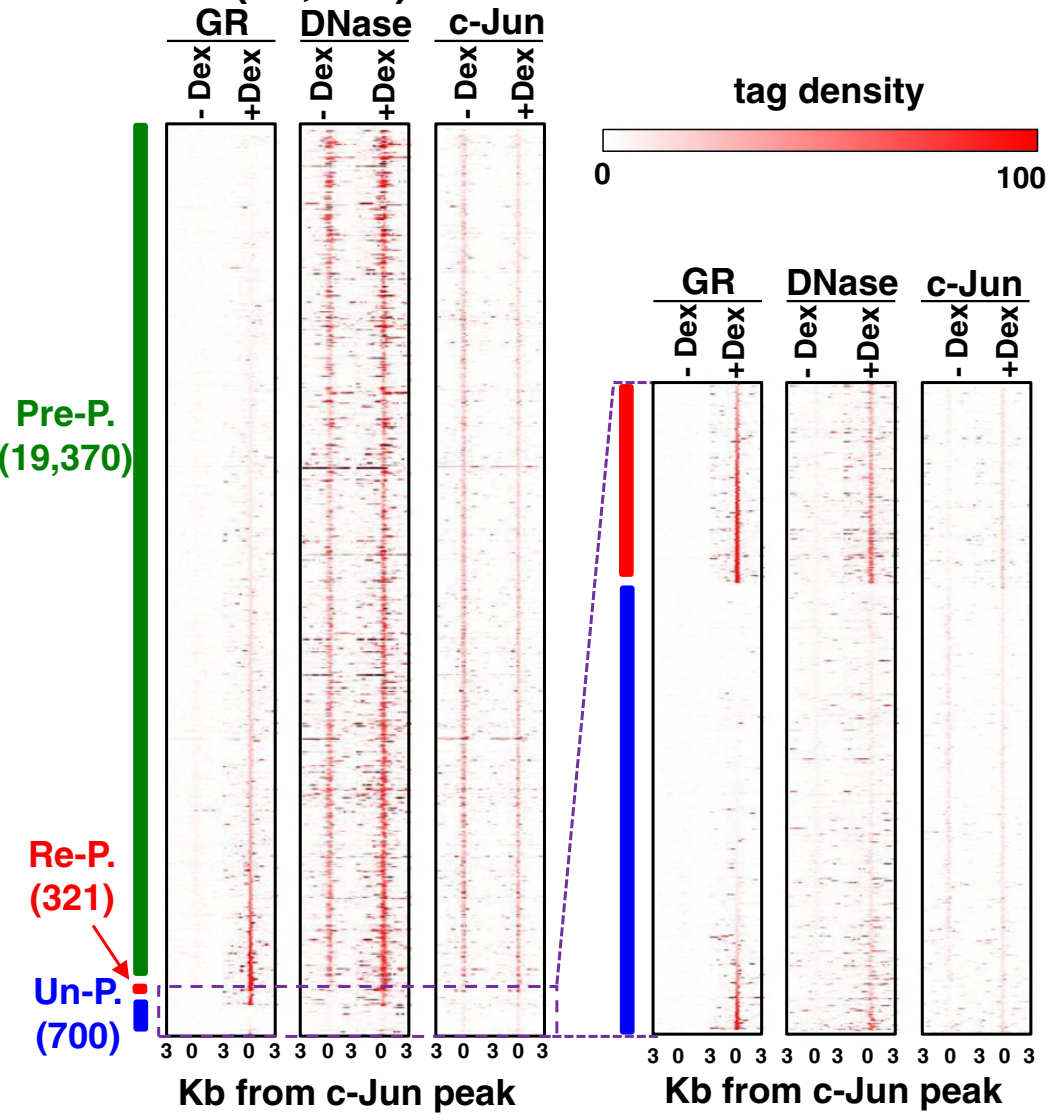

C

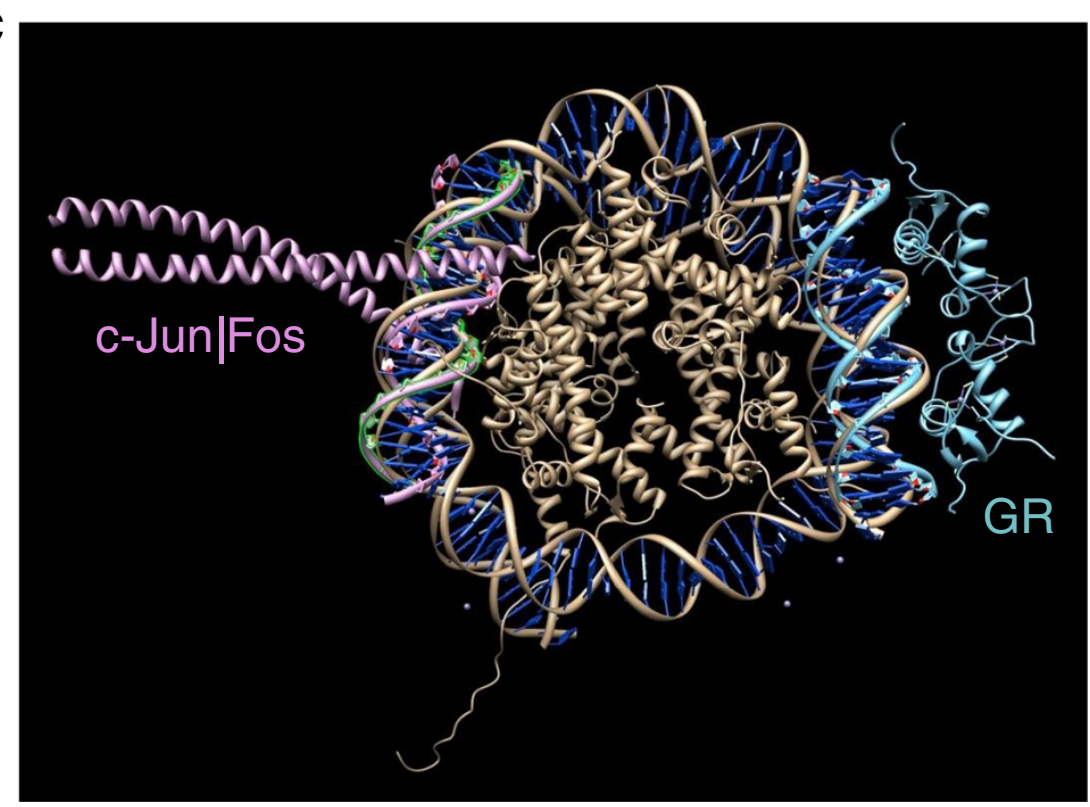

Figure $\mathbf{3}$ (See legend on next page.) 
(See figure on previous page.)

Figure 3 Chromatin accessibility of GR and AP-1 motifs in $\mathbf{3 1 3 4}$ cell line in vivo. (A-B) Density of sequenced tags for GR, C-Jun ChIP-seq and DNase I-seq were counted in 3-Kb up and downstream of the center of (A) the GR ChIP-seq peaks and (B) the C-Jun ChIP-seq peaks. GR peaks are placed in three groups, pre-programmed DHS peaks, re-programmed DHS peaks, and GR peaks that are not in a DHS peak termed as un-programmed. The bin size is 300-bps and slide window is 150-bps. Sequence reads for GR, DHS, and c-Jun are normalized w.r.t the total tag-density. Re-programmed and un-programmed c-Jun peaks are enlarged to better present the tag-density pattern. (C) Overlapping crystal structures for the nucleosome (PDB ID: 1AOI), GR (1R4O) and c-Jun|Fos (1FOS) showing GR can bind the nucleosome-occluded DNA while c-Jun|Fos has steric hindrances.

We next examined how the three classes of GR motifs at pre-, re-, and un-programmed DHSs correlate with expression of nearby genes (Additional file 1: Table S4A-C). GR peaks with canonical motifs at pre-programmed DHS tend to activate nearby genes (34\%) compared to canonical motifs in un-programmed DHS (14\%). In contrast, un-programmed peaks without a motif tend to be more activating (39\%) in comparison to the pre-programmed peaks $(21 \%)$.

\section{Clusters of identical TF motifs are better bound}

To identify additional sequence properties besides INOS that are predicative of TF binding to canonical motifs, we examined co-localization with the same sequence (homotypic clusters [32]) or a second sequence. Canonical motifs were placed into bins depending on the distance to the nearest canonical motif. Additional file 1: Figure S5A shows, for example, there are only $\sim 300$ GR motifs with a second GR within 150-bps and $18 \%$ are bound by GR. This increase relative to the $\sim 5 \%$ bound for a single GR motif may be because they are called as a single peak. In contrast, there are 1,000 GR motifs that do not have nearest neighbor within 100,000-bps and only $3 \%$ are bound (Additional file 1: Figure S5A). Similarly, $18 \%$ of the 20,000 AP-1 motifs that are within 150-bps of a second AP-1 motif are bound by c-Jun (Additional file 1: Figure S5B). Adding the clustering of GR motifs into the logistic regression increased the PVE from 3.9\% to $4.2 \%$ and AUC from 0.66 to 0.67 (Table 1, Additional file 1: Table S1B).

\section{An AP-1 motif within 150-bps of a GR motif triples GR binding}

Previously, the AP-1 motif was shown to enrich in GR peaks [21]. To identify a second sequence enriched in GR peaks, we compared the enrichment of all GR-like split 8-mers (N-NNN-NNN-N) to continuous 8-mers (NNNNNNNN) (Figure 4A). The three most enriched continuous 8-mers are AP-1 canonical motifs ( $>5$-fold enriched).

The effect of the distance between AP-1 and GR motifs on GR binding was examined (Figure 4B-D). In the masked genome, there is a decrease in the number of GR and AP-1 motif pairs as the distance between them increases from 0-bps to 900-bps (Figure 4B). There are 1,566 occurrences of an AP-1 motif within 150-bps of a GR motif in the genome, much higher than expected $\left(\mathrm{p}=3 \times 10^{-53}\right)$ calculated using in silico random sampling in the genome (Figure 4C-D). When we examined GR ChIP-seq peaks, GR motifs with a nearby AP-1 motif are better bound, with a clear inflection at 150-bps. At shorter distance, there is no unique spacing between the two motifs implying no direct physical interaction between GR and c-Jun (Additional file 1: Figure S6A) with GR and AP-1 motifs that are closer together being better bound (Figure 4B). If the two motifs are between 150bps to 900-bps, the observed occurrences in the genome and GR peaks is closer to expected occurrences. We also examined the co-occurrence of GR and AP-1 motifs in all DHS regions ( $10 \%$ of mouse genome), and the similar results are observed (Figure 4E-F). For GR motifs with an AP-1 motif within 150-bps, GR preferentially binds motifs with higher INOS (Additional file 1: Figure $\mathrm{S6B})$. The PVE and AUC by the co-occurrence of an AP-1 motif within 150-bps of a GR motif are $2.6 \%$ and 0.57 , and the addition of INOS to AP-1 co-occurrence increased the PVE to $6.7 \%$ and the AUC to 0.70 (Table 1). There are 53 GR motifs with two or more AP1 motifs within 150 -bps in the genome and $28 \%$ of them are bound suggesting that the AP-1 motifs can act additively (Figure 4G, Additional file 1: Table S1B, 5A).

The enrichment of AP-1 motifs in GR peaks occurs primarily in pre-programmed GR peaks. $66 \%$ of the pre-programmed GR peaks $(3,891 / 5,865)$ overlapped with c-Jun peaks, $13 \%$ of the re-programmed GR peaks (259/ $1,946)$ overlapped with c-Jun peaks, and only $1 \%$ of unprogrammed GR peaks (3/425) co-occur with a c-Jun peak (Figure 3A). When examined from the c-Jun perspective, $20 \%$ of the pre-programmed c-Jun peaks $(3,812 / 19,370)$ have a GR peak while $93 \%$ of re-programmed c-Jun peaks co-occur with the GR peaks (297/321) suggesting a mechanism where c-Jun promotes GR binding by creating new DHSs, and only $13 \%$ of the un-programmed c-Jun peaks co-occur with a GR peak (91/700) (Figure 3B).

\section{Bound 1-bp variants of the GR motif are more enriched for AP-1 motifs than canonical GR motifs}

Several GR-like split 8-mers are enriched in GR peaks and the 5 most enriched sequences are 1-bp variants of the canonical GR motif. The occurrence of the GR canonical motif and its 1-bp variants in the pre-, re-, and un-programmed GR peaks (Table 2, Additional file 1: Figure S7A) showed that the two canonical GR motifs and 

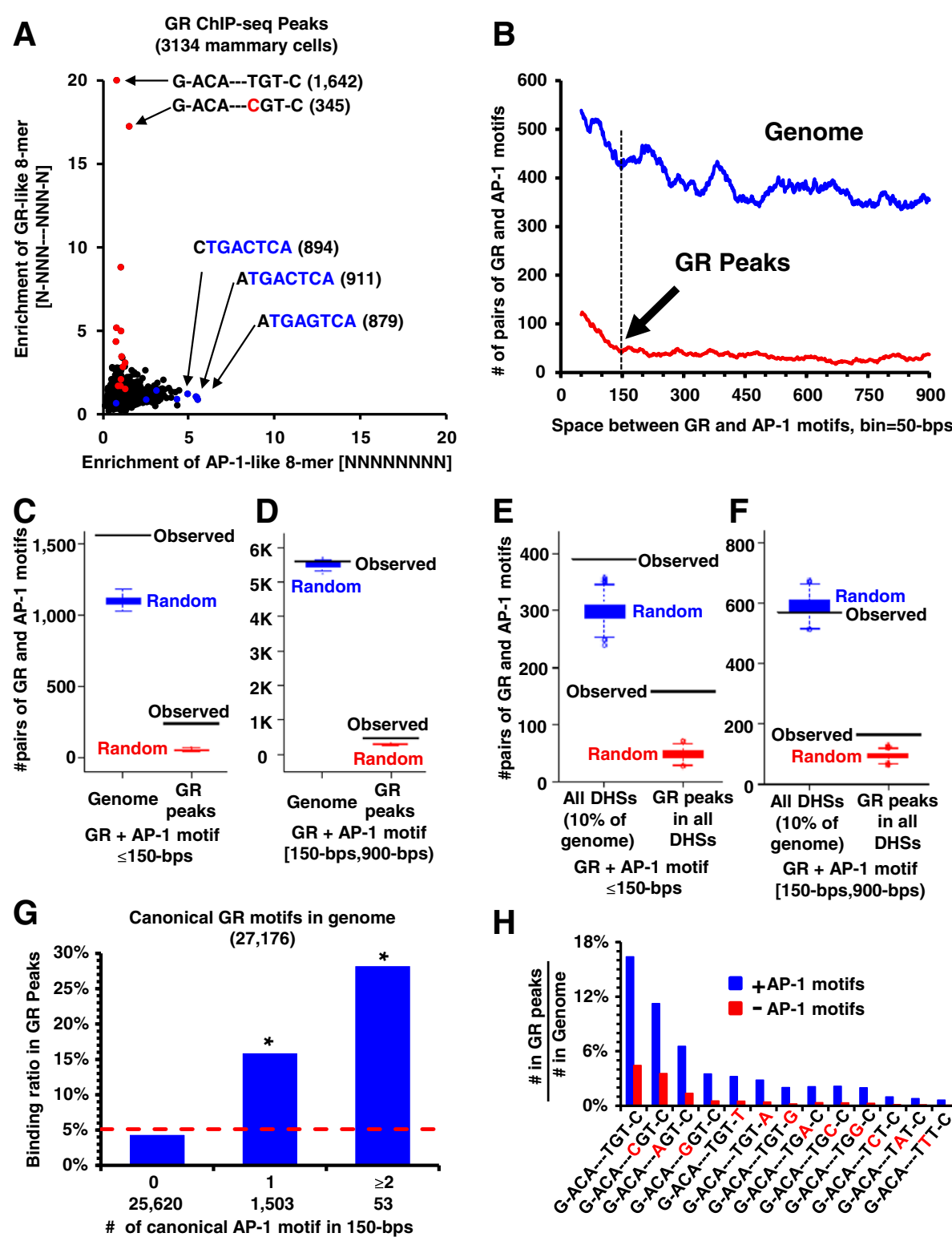

H

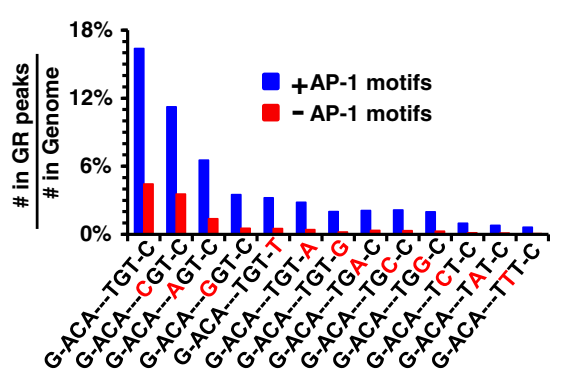

Figure 4 GR and AP-1 motifs co-occurrence in the genome and GR peaks. (A) Enrichment of split 8-mers (N-NNN-NNN-N) vs. all continuous 8mers in the GR ChIP-seq peaks. Red dots: the canonical GR motif and 1-bp variants (mismatch in red), Blue dots: the 8-mers with AP-1 motif.(B) The cooccurrence of canonical GR and AP-1 motifs at distances up to 900-bps in masked mouse genome (blue) and GR peaks (red). The bin size is 50-bps with 1-bp sliding window. (C) The co-occurrence of canonical GR and AP-1 motifs within a nucleosome range (<150-bps) is statistically significant in both the genome $\left(p=3 \times 10^{-53}\right)$ and GR peaks $\left(p=4 \times 10^{-162}\right)$. (D) Same as (C) but for GR and AP-1 motifs separated by 150-bps to 900-bps. Their cooccurrences in the genome are close to expected occurrences $(p=0.08)$ but enriched in GR peaks $\left(p=4 \times 10^{-49}\right)$. (E) The co-occurrence of canonical $G R$ and AP-1 motifs within a nucleosome range $(<150-b p s)$ is statistically significant in both the DHS regions $\left(p=2 \times 10^{-7}\right)$ and $G R$ peaks $\left(p=1 \times 10^{-179}\right)$. $(\mathbf{F})$ Same as (E) but for GR and AP-1 motifs separated by 150-bps to 900-bps. Their co-occurrences in the DHS regions are close to expected occurrences $(p=0.77)$ but enriched in GR peaks $\left(p=3 \times 10^{-12}\right)$. (G) Number of GR motifs with 0,1 , or 2 or more AP-1 motifs within 150-bps and y-axis represents percent in the GR peaks. The * denotes the statistical significance $\left(p<1 \times 10^{-10}\right)$ of co-occurring GR and AP-1 motifs being bound by GR protein compared to GR motifs without an AP-1 motif. (H) Percent of canonical or 1-bp variants of GR motifs with (blue) or without (red) canonical AP-1 motifs within 150-bps in the GR peaks. Motifs are presented by their enrichment in the GR peaks.

the 1-bp variants are more frequent in the reprogrammed and un-programmed GR peaks than the preprogrammed GR peaks. For all 1-bp variants of GR motif in the genome, we determined what percent are bound by GR and if one or more AP-1 motifs was within 150-bps.
Relaxing the GR motif of G-ACA--- ${ }^{\mathrm{T}} / \mathrm{C}$ GT-C by 1-bp decreased GR binding to less than $1 \%$ of all occurrences in the genome. However, the presence of AP-1 motifs within 150-bps to the 1-bp variants of GR motif increased GR binding by $\sim 8$-fold (Figure $4 \mathrm{H}$ ). Taken together, AP-1 
motifs contribute significantly to GR binding but preferentially to non-canonical GR motifs. Similar to the canonical AP-1 motif, 1-bp variants of the AP-1 motif can contribute to GR binding (Additional file 1: Table S1B and Table S5A-B). Co-occurrences of AP-1 motif with 1-bp variants of GR motifs are always higher than those with the canonical GR motifs in the GR ChIP-seq peaks, although their binding intensity is lower than the canonical GR motifs (Table 2, Additional file 1: Figure S7B). These data suggest a prominent role of AP-1 in creating DHS that subsequently facilitate GR binding to 1-bp GR variants in the pre-programmed DHS [22].

\section{Co-occurring AP-1 motifs need c-Jun binding for GR binding}

The contribution of c-Jun binding to GR binding was examined using A-FOS, a dominant negative protein that heterodimerizes with c-Jun and prevents DNA binding $[22,33]$. The GR peaks with more than a $50 \%$ decrease in tag-density upon A-FOS expression are considered c-Jun dependent. For the pre-programmed motifs, A-FOS expression inhibited $44 \%$ of GR binding to canonical GR motifs when there was an AP-1 motif within 150-bps but only $15 \%$ of GR binding to canonical GR motifs without a nearby canonical AP-1 motif. Similar results were observed for all 1-bp variants of GR motifs (Table 2, Additional file 1: Figure S8). A-FOS expression had similar effects on the re- and un-programmed GR peaks that contain a GR and AP-1 motif within 150-bps. The effect of A-FOS was lower in the re- and un-programmed GR peaks without an AP-1 motif again supporting the observation that presence of nearby AP-1 motif helps create a DHS facilitating GR binding to the canonical and 1-bp variants of GR motifs.

\section{GR binds different GR motifs in AtT-20 cells}

In a mouse pituitary AtT-20 cell line, GR bound different canonical GR motifs that also have high INOS (Figure 5A-B), suggesting that this trait is a general principle regulating GR localization (Additional file 1: Table S1B). The logistic regression analysis indicates that the PVE and AUC of INOS in peaks for GR localization are $2.6 \%$ and 0.65 in AtT-20 cells (Additional file 1: Table S1B). Instead of co-occurrence with AP-1 motifs (Figure 5C), GR co-occurred with the E-Box motif (CAGCTGT) in AtT-20 cells (Figure 5C-E, Additional file 1: Table S5C) suggesting that the co-occurrence of a second TFBS might be a cell type dependent mechanism. The addition of nearby E-box motif to the logistic regression increased the PVE for GR binding from $2.6 \%$ to $3.5 \%$ (Additional file 1: Table S1B).

\section{Discussion}

The advent of the ChIP-seq technique [34-36] has produced detailed maps of transcription factor binding in the genome. These data sets are often used to produce a position weight matrix for preferred binding sites. However, it is difficult to compare the properties of one TF with another. We have used a logistic regression to evaluate sequence properties near TFBS that are predicative of a TFBS being bound in vivo. We examined the INOS of TFBS embedded in 150-bps of DNA and the cooccurrence of a secondary motif. These descriptors are different for the three TFs examined, GR, c-Jun, and Hoxa2 binding the GR, AP-1 and Pbx motifs respectively.

Recent computational models for calculating the strength of the in vitro binding of a nucleosome to any 150-bp length of DNA $[4,5,37]$ allows evaluation of the significance of this parameter to TF localization in the genome. Many TFs bind in regions that have higher INOSs than neighboring DNA and are depleted for nucleosomes [5] [30] suggesting a competition model of gene regulation [18]. We determined the INOS for each canonical GR, AP-1, and Pbx motif in the genome and identified which ones were bound by examining ChIPseq data for GR and c-Jun. Our analyses showed that both GR and c-Jun bind preferentially to the canonical motifs predicted to be well bound by nucleosomes (Figure 1) lending support to the competition model of gene regulation [18] that we were able to quantify using a logistic regression. These data indicate that high INOS is more predictive of c-Jun localization (PVE $=11.5$, and AUC $=0.77)$ than GR localization (PVE $=3.9$, and AUC $=0.66)$ with $\mathrm{Pbx}$ showing little localization dependent on high INOS (PVE $=0.6$, and AUC $=0.57$ ). The greater value for c-Jun localization to AP-1 motifs than GR binding to GR motifs suggest c-Jun may be more involved in competitive interactions with nucleosomes, such as maintaining or creating DHS [22]. Both GR and Pbx binding motifs with low INOS suggesting a second mode of function for these two proteins. The significance of these differences observed among GR, c-Jun and Hoxa2 using the logistic regression will become clearer as additional TFs are examined. More accurate models of sequence specific nucleosome binding will improve the accuracy of these predictions.

Competition models for TF and nucleosome binding the same sequence have been extended to collaborative competition models where two TFs can bind to DNA independently but on their own do not displace a nucleosome. However, cooperatively they displace a nucleosome if they are within 150-bps [10,11,15-18]. This switch mechanism is observed in mammalian genomes $[3,7]$ where regulatory regions [1] are often CpG islands that are well bound by nucleosomes in vitro [8] but not in vivo. The mechanism for the switch is complex. For example, some TFs (e.g. GR) can bind to a motif that is also bound by the histone octamer, while other TFs (e.g. c-Jun) can only bind to motif only when the histone 


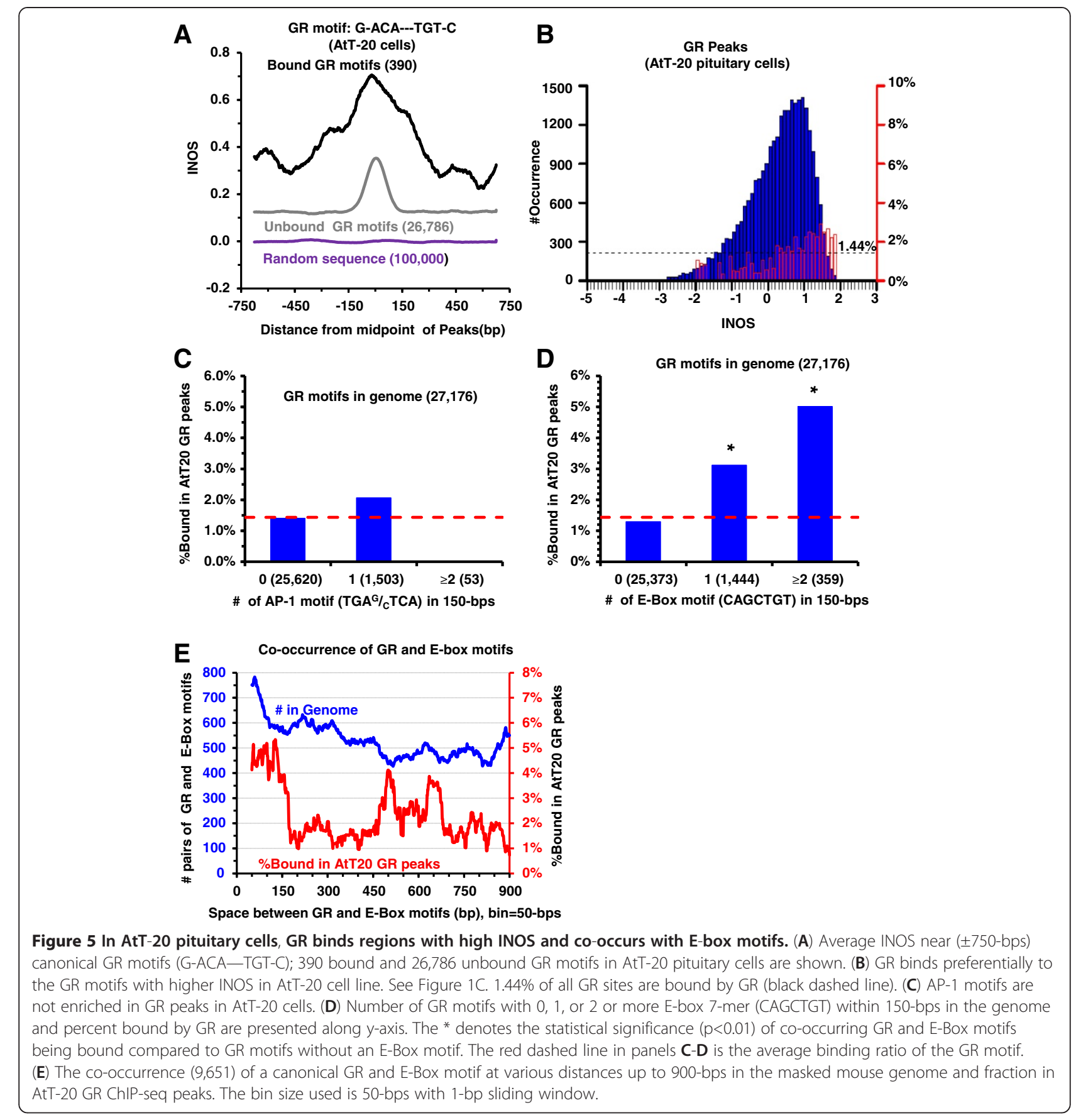

octamer is not bound. Additional cooperativity to the switch can be achieved with cooperative binding of TF to their TFBSs $[28,38]$.

The ability of two TFs to compete with a nucleosome for binding suggests that they are within 150-bps of each other [18]. When we examine the entire genome, there are $~ 1,500$ occurrences of GR and AP-1 motifs within 150-bps, compared to 1,100 occurrences expected, indicating that co-occurring GR and AP-1 motifs are enriched in the genome. In the mammalian genome, the clustering of TFBS is always observed when the two TFBS contain a CG dinucleotide [9] because of the presence of $\mathrm{CpG}$ islands in the genome. However, neither the abundant GR motif (G-ACA---TGT-C) nor the AP-1 motif contains a CG dinucleotide suggesting their preferential localization in the genome is independent from the clustering of TFBS containing CG. For the rare CG dinucleotide containing GR motif (G-ACA---CGT-C), the methylation status of the CG can regulate function adding a layer of complexity onto GR activity [39]. 
The collaborative competition model allows a single TF to interact with different TF in different cell types producing multiple functions. This is consistent with the observations concerning GR co-localization with different DNA motifs in the two cell types examined. In the mouse pituitary cell line AtT-20, GR binds GR motifs that have high INOS. But instead of being enriched in AP-1 motifs, GR binds canonical motifs enriched in the co-localizing E-Box motif that are within 150-bps. GR binds preferentially to motifs with high INOS in both 3134 and AtT-20 cells. Thus, the propensity to bind a nucleosome may be a general parameter in determining GR localization. However, the nucleosome may be displaced by different collaborative TFs in difference cells, suggest that nucleosome positioning can be cell-specific.

TFBS are often clustered in regulatory regions $[18,32,40,41]$ where low-affinity TFBSs may play a role in assisting high-affinity motifs bind a $\mathrm{TF}(\mathrm{s})$ to displace a nucleosome $[11,42] .83 \%$ of GR peaks and $42 \%$ of c-Jun peaks do not contain canonical motifs. However, many 1-bp variants are enriched in GR and c-Jun peaks, allowing us to survey the difference of collaborative competition for canonical and 1-bp variants. We observed that a canonical AP-1 motif facilitates GR binding to 1-bp variants ( 8 folds) better than the two canonical GR motifs ( $\sim 3$ folds).

The intrinsic nucleosome occupancy is correlated with GC content, which implies that some TFBSs with high GC content may be well bound by nucleosomes [4,5,8,37], while others with low GC content may be not. Both GR and AP-1 canonical motifs are calculated to be well bound by nucleosomes. The GR (G-ACA---TGT-C) and AP-1 (TGA ${ }^{\mathrm{C}} / \mathrm{G}_{\mathrm{TCA}}$ ) motifs have two pyrimidine-purine dinucleotides (in bold) separated by 5 -bps. These sequences wrap well around a nucleosome, which may be the reason why nucleosomes are calculated to bind these sequences well [43]. Besides GR and AP-1 motifs, many additional 8mers, such as the CRE (TGACGTCA) and CTCF motif (AGGGGGCG) have a high INOS, which indicates that many TFs bind to the same sequences calculated to be well-bound by the nucleosome and produce an intrinsic competition between TF and nucleosomes for binding the same DNA. In contrast, some motifs, e.g., Pbx (TGATTGAT) bound by Hoxa2 are not well bound by nucleosomes suggesting they are not competing for binding to the same DNA. Hoxa2 ChIP-seq data showed binding to motifs with both high and low INOS suggesting that this protein can function in two separate mechanisms. These results are consistent with what Charoensawan et. al recently observed in yeast [29].

Examples of a non-competitive model for TF and nucleosome binding are observed in yeast. Generally, yeast promoters are AT-rich with lower nucleosome occupancy both in vitro and in vivo [4,44-46]. A recent study showed that, in yeast the transcriptional activators with high intrinsic nucleosome binding properties might compete with nucleosomes, while the repressors are intrinsically less likely to compete with nucleosomes [29]. In yeast, TFs can recognize a specific regulatory region in the genome background to regulate gene expression, while in higher eukaryotes, such as human and mouse, TFBSs must be clustered to achieve specificity and collaborate to compete with nucleosomes [2].

\section{Conclusion}

We have used a logistic regression to quantify the contribution of INOS and co-occurring sequence to TF binding in the genome. This strategy will allow investigators to more richly compare the properties of different TFs. Only AP-1 motifs with high INOS were preferentially bound while GR and Hoxa2 bound canonical motifs with both high and low INOS suggesting these proteins can function using two mechanisms.

\section{Methods \\ Data sets}

The reference genome of mouse (masked and unmasked, $\mathrm{mm} 9$ ) and DHS peaks in 55 samples from ENCODE project [47] are obtained from University of California Santa Cruz Genome Bioinformatics website (http://genome.ucsc. edu/) $[48,49]$. The data of GR ChIP-seq peaks, DHS peaks and expression array data in mouse 3134 mammary cells and pituitary cell line AtT-20 is from previous study [21] deposited at NCBI with SRA number of SRP004871 and GEO number of GSE26189. The data of c-Jun ChIP-seq peaks and GR ChIP-seq peaks upon A-FOS is from the study [22] deposited at NCBI with SRA number of SRP007111. The data of Hoxa2 ChIP-seq peaks is downloaded from supplementary data of Donaldson et. al's study [23] deposited at NAR Online. The canonical motifs for GR, AP-1 and Pbx are selected based on the enrichment of GR-like 8-mers, 7-mers, 8-mers in GR, c-Jun and Hoxa2 ChIP-seq peaks. Custom Perl scripts are used to search GR, AP-1 and Pbx motifs base by base across the whole masked mouse genome of $\mathrm{mm} 9$, and to extract the sequences with 750 -bps upstream and downstream to the center of each peak and motif from the unmasked genome of $\mathrm{mm} 9$.

\section{Enrichment of GR-like 8-mers and AP-1 like 8-mers in ChIP-seq peaks}

To calculate the enrichment of different 8-mers, we first generated the unique 32,8968 -mers by ignoring the complementary reverse 8 -mers. Then we extracted the sequences with 750-bps upstream and downstream to the center of each ChIP-seq peak from the unmasked genome of $\mathrm{mm} 9$. For each sequence, we defined the DNA fragment of \pm 150 -bps to the center of peak as Peak region, and \pm 750 -bps to \pm 150 -bps to the center of peak as Background region. For each 8-mer, we count 
the occurrence of the 8-mer in Peak region as \#PK, and occurrence of the 8-mer in Background region as \#BG. The enrichment for each 8 -mer $\left(\mathrm{E}_{8 \text {-mer }}\right)$ is then calculated as: $\mathrm{E}_{8-\mathrm{mer}}=\frac{\# P K}{\# B G} \times\left(\frac{1500-300}{300}\right)$.

\section{Intrinsic nucleosome occupancy calculation}

Two models for calculation of intrinsic nucleosome occupancy are used in our analysis. One is intrinsic nucleosome occupancy score (INOS) based on Lasso algorithm from Hughes' group [5,37] and the second model to predict nucleosome occupancy probability (PNOP) uses Segal's model [4]. For each 1,500-bps sequence, we calculated the INOSs and PNOPs for each 147-bps slide window and moved the window one base-pair at a time to get the profile of INOSs and PNOPs. The control set is 100,000 sequences random selected from mm9. The Peak value of INOS is calculated from the middle 147-bps of each peak or motif. The Background value of INOS is the average INOSs of regions from \pm 750 to \pm 150 -bps to the peak or motif. The Relative Peak value of INOS is calculated as Peak value minus Background value. The INOS for excluding a specific motif (G-ACA-TGT-C or $\mathrm{TGA}^{\mathrm{C}} / \mathrm{G}_{\mathrm{G}} \mathrm{TC}$ ) is calculated from the sequence whose motif is replaced by random bases but with GC content of $42 \%$ as in the mouse genome.

\section{Modeling GR and c-Jun binding by a logistic regression}

To analyze the GR and c-Jun binding to the canonical motifs, we performed a logistic regression using the generalized linear model (GLM) with the R statistical language. GLMs were formulated by John Nelder and Robert Wedderburn as a way of unifying various other statistical models, including linear regression, logistic regression and Poisson regression [50]. GLM is a standard package in $\mathrm{R}$ language for computation and modeling. For each motif $M_{i}$, the binding value $\left(B V_{i}\right)$ for $M_{i}$ is 1 if motif $M_{i}$ occurs in the ChIP-seq peaks, otherwise BV $_{i}$ is 0 . Three INOSs for each $M_{i}$ are used for evaluated parameters: INOS of the Peak denoted as INOS ${ }^{\mathrm{p}}$, INOS of the Backgroud denoted as INOS ${ }^{\mathrm{b}}$, INOS of the Relative Peaks denoted as INOS $^{\text {rp }}$. For the parameters of overlap with CGIs (CGI), with in clusters (CLT), co-occurrence with the second motifs (CO), and located in DHSs (DHS), if it is true, the value is 1 , otherwise is 0 . The formula for calculated the GLM in R is: BV $\sim \mathrm{INOS}^{\mathrm{p}}+\mathrm{INOS}^{\mathrm{b}}+\mathrm{INOS}^{\mathrm{rp}}+\mathrm{CGI}+$ $\mathrm{CLT}+\mathrm{CO}+\mathrm{DHS}$, with the binomial distribution. Let $\mathrm{P}_{\mathrm{BV}}$ be the conditional probability of motif $M_{i}$ being bound, which is generated from the independent variables of INOS $^{\mathrm{p}}, \mathrm{INOS}^{\mathrm{b}}$,INOS ${ }^{\mathrm{rp}}, \mathrm{CGI}, \mathrm{CLT}, \mathrm{CO}$, and DHS: $\mathrm{P}_{\mathrm{BV}}=\mathrm{P}$ $\left\{B V=1 \mid\right.$ INOS $^{p}$, INOS $^{b}$, INOS $^{\mathrm{rp}}, C$ CGI, CLT, CO, DHS $\}$, then the logistic regression is:
Where $\beta_{0}$ is constant, and $\beta_{1}, \beta_{2}, \beta_{3}, \beta_{4}, \beta_{5}, \beta_{6}$, and $\beta_{7}$ are the coefficients for INOS $^{\mathrm{p}}$, INOS ${ }^{\mathrm{b}}, \mathrm{INOS}^{\mathrm{rp}}$, CGI, CLT, CO, and DHS respectively. By logit transformation (link function), a linear regression is generalized from formula (1), as follows:

$$
\begin{aligned}
\operatorname{logit}\left(\mathrm{P}_{\mathrm{BV}}\right)= & \ln \left[\mathrm{P}_{\mathrm{BV}} /\left(1-\mathrm{P}_{\mathrm{BV}}\right)\right] \\
= & \beta_{0}+\beta_{1} \times \mathrm{INOS}^{\mathrm{P}}+\beta_{2} \times \mathrm{INOS}^{\mathrm{b}} \\
& +\beta_{3} \times \mathrm{INOS}^{\mathrm{rp}}+\beta_{4} \times \mathrm{CGI}+\beta_{5} \\
& \times \mathrm{CLT}+\beta_{6} \times \mathrm{CO}+\beta_{7} \times \mathrm{DHS}
\end{aligned}
$$

The percent of variance explained (PVE) is calculated as: $\mathrm{PVE}=(1-($ deviance $/$ null.deviance $)) \times 100$. For each parameter, the PVE denotes the significance for predicting GR or c-Jun binding: the higher value of PVE means the parameter is more predictive. We also estimated area under the ROC curve (AUC) using 11-fold cross-validation to measure the predictive ability of the logistic regression model for comparison between TFs.

\section{Calculation of INOS for GR-like 8-mers and AP-1-like 8- mers}

To calculate the INOS for each 8-mer, either GR-like (N-NNN-NNN-N) or AP-1-like (NNNNNNNN), we first simulated a 150-bps DNA sequence using Markov model with the 8-mer fixed in the center and with the same GC content (42\%) as mouse genome. We used the seventh-order Markov model to produce the simulated 150 bps. The DNA sequences were generated by using the 8-mer frequencies observed in mouse genome. To populate each 150-bps DNA sequence, initially an 8-mer was chosen at random. To determine each next base, the preceding 7-mer was identified. The frequency of the four 8-mers starting with this 7-mer was determined, and the next base-pair was chosen by chance maintaining this frequency. This process was continued until the entire 150-bps sequence was determined. Then we calculated the INOS for the 150-bps DNA sequence. For each 8-mer, we repeat the simulation for 1,000 times and the average value of the 1,000 INOSs is treated as the INOS for the 8mer. A random control set of DNA sequences are also calculated with all 150-bps are simulated using Markov model with GC content of $42 \%$. For each control 8-mer, 1,000 sequences 150 -bps long are simulated, and 32,896 times are repeated to get the whole random control set.

\section{Simulation of co-occurrence of GR and AP-1 canonical motifs}

To simulate the co-occurrence of GR and AP-1 canonical motifs in the genome, we used uniform location

$$
\mathrm{P}_{\mathrm{BV}}=\frac{\exp \left(\beta_{0}+\beta_{1} \times \mathrm{INOS}^{\mathrm{p}}+\beta_{2} \times \mathrm{INOS}^{\mathrm{b}}+\beta_{3} \times \mathrm{INOS}^{\mathrm{rp}}+\beta_{4} \times \mathrm{CGI}+\beta_{5} \times \mathrm{CLT}^{2} \beta_{6} \times \mathrm{CO}+\beta_{7} \times \mathrm{DHS}\right)}{1+\exp \left(\beta_{0}+\beta_{1} \times \mathrm{INOS}^{\mathrm{p}}+\beta_{2} \times \mathrm{INOS}^{\mathrm{b}}+\beta_{3} \times \mathrm{INOS}^{\mathrm{rp}}+\beta_{4} \times \mathrm{CGI}+\beta_{5} \times \mathrm{CLT}+\beta_{6} \times \mathrm{CO}+\beta_{7} \times \mathrm{DHS}\right)}
$$


model: the same occurrences of GR and AP-1 canonical motifs are generated in each masked chromosome, but each location of the motif is selected uniformly at random from each masked chromosome. For each chromosome with length $\mathrm{N}$, we first generated the locations where bases are not Ns $(A|C| T \mid G)$, as $\{X+1, X+2, X+n\}$, $X \in 1, \ldots N$. If the canonical motif occurs in the chromosome $M$ times, then we random selected $M$ positions from $\{\mathrm{X}+1, \mathrm{X}+2, \ldots, \mathrm{X}+\mathrm{n}\}$ as the simulated occurrence. Then we simulated for all the 22 chromosomes to get the whole genome simulation. After generating the simulated occurrence of GR and AP-1 canonical motifs, we calculated the distance between the two motifs. 1,000 same simulations are repeated to generate the distribution of co-occurrence of simulated GR and AP-1 motifs with 150-bps and from 150-bps to 900-bps. Similarly, we simulated co-occurrence of the GR and AP-1 motifs in the DHS regions ( $10 \%$ genome), where all the simulated GR and AP-1 motifs occurred only in the DHS regions.

\section{Additional file}

\section{Additional file 1: Supplementary Figures and Tables.}

\section{Abbreviations}

TF: Transcription factor; TFBS: Transcription factor binding site; GLM: Generalized linear model; INOS: Intrinsic nucleosome occupancy score; DHS: DNase I hypersensitive sites; PVE: Percent of variance explained; PNOP: Predicted nucleosome occupancy probability; GR: Glucocorticoid receptor; Hoxa2: Homeobox a2; Pbx: Pre-B-cell leukemia homeobox; AUC: Area under the ROC curve.

\section{Competing interests}

The authors have no competing interests to declare.

\section{Authors' contributions \\ $\mathrm{XH}, \mathrm{RC}, \mathrm{BKS}$ performed the computational analyses. SJ and SCB conducted the experiments. XH, HB, PCF, JAS, GLH and CV conceived of the study. XH drafted the manuscript. RC and CV helped to revise the manuscript. All authors read and approved the final manuscript.}

\section{Acknowledgments}

We thank Dr. Jianfei Zhao, Lars Grontved, and Myong-Hee Sung of NCl, NIH for their thoughtful comments and high-performance computational capabilities of the Helix \& Biowulf Systems at the NIH, Bethesda, MD (http://helix.nih.gov).

\footnotetext{
Author details

'Laboratory of Metabolism, National Cancer Institute, National Institutes of Health, Room 3128, Building 37, 37 Convent Drive, Bethesda, MD 20892, USA. ${ }^{2}$ Laboratory of Receptor Biology and Gene Expression, National Cancer Institute, National Institutes of Health, B602, Building 41, 41 Library Drive, Bethesda, MD 20892, USA. ${ }^{3}$ Laboratory of Molecular Biology, National Cancer Institute, National Institutes of Health, Bethesda, MD 20892, USA. ${ }^{4}$ Genome Analysis Unit, Genetics Branch, National Cancer Institute, National Institutes of Health, Bethesda, MD 20892, USA. ${ }^{5}$ Center for Bioinformatics and Computational Biology, Biomolecular Sciences Bldg \#296, University of Maryland, College Park, MD 20742, USA. ${ }^{6}$ Department of Genome Sciences, University of Washington, Seattle, WA 98195, USA.
}

Received: 16 January 2013 Accepted: 10 June 2013

Published: 28 June 2013

\section{References}

1. FitzGerald PC, Shlyakhtenko A, Mir AA, Vinson C: Clustering of DNA sequences in human promoters. Genome Res 2004, 14:1562-1574.

2. Wunderlich Z, Mirny LA: Different gene regulation strategies revealed by analysis of binding motifs. Trends Genet 2009, 25:434-440.

3. FitzGerald PC, Sturgill D, Shyakhtenko A, Oliver B, Vinson C: Comparative genomics of Drosophila and human core promoters. Genome Biol 2006, 7:R53.

4. Kaplan N, Moore IK, Fondufe-Mittendorf Y, Gossett AJ, Tillo D, Field Y, LeProust EM, Hughes TR, Lieb JD, Widom J, Segal E: The DNA-encoded nucleosome organization of a eukaryotic genome. Nature 2009, 458:362-366.

5. Tillo D, Kaplan N, Moore IK, Fondufe-Mittendorf Y, Gossett AJ, Field Y, Lieb JD, Widom J, Segal E, Hughes TR: High nucleosome occupancy is encoded at human regulatory sequences. PLoS One 2010, 5:e9129.

6. Kaplan N, Hughes TR, Lieb JD, Widom J, Segal E: Contribution of histone sequence preferences to nucleosome organization: proposed definitions and methodology. Genome Biol 2010, 11:140.

7. Vinson C, Chatterjee R, Fitzgerald P: Transcription factor binding sites and other features in human and Drosophila proximal promoters. subcell Biochem 2011, 52:205-222.

8. Valouev A, Johnson SM, Boyd SD, Smith CL, Fire AZ, Sidow A: Determinants of nucleosome organization in primary human cells. Nature 2011, 474:516-520.

9. Rozenberg JM, Shlyakhtenko A, Glass K, Rishi V, Myakishev MV, FitzGerald $P C$, Vinson C: All and only $\mathrm{CpG}$ containing sequences are enriched in promoters abundantly bound by RNA polymerase II in multiple tissues. BMC Genomics 2008, 9:67.

10. Polach KJ, Widom J: Mechanism of protein access to specific DNA sequences in chromatin: a dynamic equilibrium model for gene regulation. J Mol Biol 1995, 254:130-149.

11. Mirny LA: Nucleosome-mediated cooperativity between transcription factors. Proc Natl Acad Sci USA 2010, 107:22534-22539.

12. Bai L, Ondracka A, Cross FR: Multiple sequence-specific factors generate the nucleosome-depleted region on CLN2 promoter. Mol Cell 2011, 42:465-476.

13. Richard-Foy H, Hager GL: Sequence-specific positioning of nucleosomes over the steroid-inducible MMTV promoter. EMBO J 1987, 6:2321-2328.

14. Fedor MJ, Lue NF, Kornberg RD: Statistical positioning of nucleosomes by specific protein-binding to an upstream activating sequence in yeast. J Mol Biol 1988, 204:109-127.

15. Taylor IC, Workman JL, Schuetz TJ, Kingston RE: Facilitated binding of GAL4 and heat shock factor to nucleosomal templates: differential function of DNA-binding domains. Genes Dev 1991, 5:1285-1298.

16. Adams CC, Workman JL: Binding of disparate transcriptional activators to nucleosomal DNA is inherently cooperative. Mol Cell Biol 1995, 15:1405-1421.

17. Polach KJ, Widom J: A model for the cooperative binding of eukaryotic regulatory proteins to nucleosomal target sites. J Mol Biol 1996, 258:800-812.

18. Miller JA, Widom J: Collaborative competition mechanism for gene activation in vivo. Mol Cell Biol 2003, 23:1623-1632.

19. Perlmann T, Wrange O: Specific glucocorticoid receptor binding to DNA reconstituted in a nucleosome. EMBO J 1988, 7:3073-3079.

20. John S, Sabo PJ, Johnson TA, Sung MH, Biddie SC, Lightman SL, Voss TC, Davis SR, Meltzer PS, Stamatoyannopoulos JA, Hager GL: Interaction of the glucocorticoid receptor with the chromatin landscape. Mol Cell 2008, 29:611-624

21. John S, Sabo PJ, Thurman RE, Sung MH, Biddie SC, Johnson TA, Hager GL, Stamatoyannopoulos JA: Chromatin accessibility pre-determines glucocorticoid receptor binding patterns. Nat Genet 2011, 43:264-268.

22. Biddie SC, John S, Sabo PJ, Thurman RE, Johnson TA, Schiltz RL, Miranda TB, Sung MH, Trump S, Lightman SL, et al: Transcription factor AP1 potentiates chromatin accessibility and glucocorticoid receptor binding Mol Cell 2011, 43:145-155.

23. Donaldson IJ, Amin S, Hensman JJ, Kutejova E, Rattray M, Lawrence N, Hayes A, Ward CM, Bobola N: Genome-wide occupancy links Hoxa2 to Wnt-beta-catenin signaling in mouse embryonic development. Nucleic Acids Res 2012, 40:3990-4001.

24. Sekinger EA, Mogtaderi Z, Struhl K: Intrinsic histone-DNA interactions and low nucleosome density are important for preferential accessibility of promoter regions in yeast. Mol Cell 2005, 18:735-748.

25. Bailey TL, Boden M, Buske FA, Frith M, Grant CE, Clementi L, Ren J, Li WW, Noble WS: MEME SUITE: tools for motif discovery and searching. Nucleic Acids Res 2009, 37:W202-W208. 
26. Jurka J: Repbase update: a database and an electronic journal of repetitive elements. Trends Genet 2000, 16:418-420.

27. Meijsing SH, Pufall MA, So AY, Bates DL, Chen L, Yamamoto KR: DNA binding site sequence directs glucocorticoid receptor structure and activity. Science 2009, 324:407-410

28. Chatterjee R, Zhao J, He X, Shlyakhtenko A, Mann I, Waterfall JJ, Meltzer P, Sathyanarayana BK, Fitzgerald PC, Vinson C: Overlapping ETS and CRE Motifs ((G)/(C)CGGAAGTGACGTCA) Preferentially Bound by GABPalpha and CREB Proteins. G3 (Bethesda) 2012, 2:1243-1256.

29. Charoensawan V, Janga SC, Bulyk ML, Babu MM, Teichmann SA: DNA sequence preferences of transcriptional activators correlate more strongly than repressors with nucleosomes. Mol Cell 2012, 47:183-192.

30. Lidor Nili E, Field Y, Lubling Y, Widom J, Oren M, Segal E: p53 binds preferentially to genomic regions with high DNA-encoded nucleosome occupancy. Genome Res 2010, 20:1361-1368.

31. Pettersen EF, Goddard TD, Huang CC, Couch GS, Greenblatt DM, Meng EC, Ferrin TE: UCSF Chimera-a visualization system for exploratory research and analysis. J Comput Chem 2004, 25:1605-1612.

32. Gotea V, Visel A, Westlund JM, Nobrega MA, Pennacchio LA, Ovcharenko I: Homotypic clusters of transcription factor binding sites are a key component of human promoters and enhancers. Genome Res 2010, 20:565-577.

33. Olive M, Krylov D, Echlin DR, Gardner K, Taparowsky E, Vinson C: A dominant negative to activation protein-1 (AP1) that abolishes DNA binding and inhibits oncogenesis. J Biol Chem 1997, 272:18586-18594.

34. Valouev A, Johnson DS, Sundquist A, Medina C, Anton E, Batzoglou S, Myers RM, Sidow A: Genome-wide analysis of transcription factor binding sites based on ChIP-Seq data. Nat Methods 2008, 5:829-834.

35. Kharchenko PV, Tolstorukov MY, Park PJ: Design and analysis of ChIP-seq experiments for DNA-binding proteins. Nat Biotechnol 2008, 26:1351-1359.

36. Furey TS: ChIP-seq and beyond: new and improved methodologies to detect and characterize protein-DNA interactions. Nat Rev Genet 2012, 13:840-852.

37. Tillo D, Hughes TR: G+C content dominates intrinsic nucleosome occupancy. BMC Bioinforma 2009, 10:442.

38. Martinez GJ, Rao A: Immunology. Cooperative transcription factor complexes in control. Science 2012, 338:891-892.

39. Wiench M, John S, Baek S, Johnson TA, Sung MH, Escobar T, Simmons CA, Pearce KH, Biddie SC, Sabo PJ, et al: DNA methylation status predicts cell type-specific enhancer activity. EMBO J 2011, 30:3028-3039.

40. Schroeder MD, Pearce M, Fak J, Fan H, Unnerstall U, Emberly E, Rajewsky N, Siggia ED, Gaul U: Transcriptional control in the segmentation gene network of Drosophila. PLOS Biol 2004, 2:E271.

41. Berman BP, Pfeiffer BD, Laverty TR, Salzberg SL, Rubin GM, Eisen MB, Celniker SE: Computational identification of developmental enhancers: conservation and function of transcription factor binding-site clusters in Drosophila melanogaster and Drosophila pseudoobscura. Genome Biol 2004, 5:R61.

42. Zhang C, Xuan Z, Otto S, Hover JR, McCorkle SR, Mandel G, Zhang MQ: A clustering property of highly-degenerate transcription factor binding sites in the mammalian genome. Nucleic Acids Res 2006, 34:2238-2246.

43. Sahu G, Wang D, Chen CB, Zhurkin VB, Harrington RE, Appella E, Hager GL, Nagaich AK: p53 binding to nucleosomal DNA depends on the rotational positioning of DNA response element. J Biol Chem 2010, 285:1321-1332.

44. loshikhes IP, Albert I, Zanton SJ, Pugh BF: Nucleosome positions predicted through comparative genomics. Nat Genet 2006, 38:1210-1215.

45. Field Y, Kaplan N, Fondufe-Mittendorf Y, Moore IK, Sharon E, Lubling Y, Widom J, Segal E: Distinct modes of regulation by chromatin encoded through nucleosome positioning signals. PLOS Comput Biol 2008, 4:e1000216.

46. Tirosh I, Barkai N: Two strategies for gene regulation by promoter nucleosomes. Genome Res 2008, 18:1084-1091.

47. Stamatoyannopoulos JA, Snyder M, Hardison R, Ren B, Gingeras T, Gilbert DM, Groudine M, Bender M, Kaul R, Canfield T, et al: An encyclopedia of mouse DNA elements (Mouse ENCODE). Genome Biol 2012, 13:418.
48. Meyer LR, Zweig AS, Hinrichs AS, Karolchik D, Kuhn RM, Wong M, Sloan CA, Rosenbloom KR, Roe G, Rhead B, et al: The UCSC Genome Browser database: extensions and updates 2013. Nucleic Acids Res 2013, 41:D64-9.

49. Kuhn RM, Haussler D, Kent WJ: The UCSC genome browser and associated tools. Brief Bioinform 2013, 14:144-61.

50. Nelder JA, Wedderbu RW: Generalized Linear Models. Journal of the Royal Statistical Society Series a-General 1972, 135:370-384.

doi:10.1186/1471-2164-14-428

Cite this article as: He et al:: Contribution of nucleosome binding preferences and co-occurring DNA sequences to transcription factor binding. BMC Genomics 2013 14:428.

\section{Submit your next manuscript to BioMed Central and take full advantage of:}

- Convenient online submission

- Thorough peer review

- No space constraints or color figure charges

- Immediate publication on acceptance

- Inclusion in PubMed, CAS, Scopus and Google Scholar

- Research which is freely available for redistribution

Submit your manuscript at www.biomedcentral.com/submit
C) Biomed Central 